\title{
Evaluación de la salud uterina por citología endometrial y ultrasonografía en el posparto y su relación con la concepción en alpacas
}

\author{
Evaluation of uterine health by endometrial cytology and ultrasonography in the \\ postpartum and its relationship with conception in alpacas
}

\author{
Hugo W. Deza ${ }^{1,4}$, Gloria E. Mamani², Rassiel Macedo ${ }^{2}$, \\ Rolando G. Alencastre², Carlos A. Gomez ${ }^{3}$
}

\section{Resumen}

\begin{abstract}
El objetivo del presente estudio fue evaluar la salud uterina de alpacas por citología endometrial (CE) mediante las técnicas de citocepillo y lavado uterino, así como evaluar las características uterinas por ultrasonografía (US) y relacionarlas con la tasa de preñez. Se utilizaron 20 alpacas multíparas y 20 primíparas, de la raza Suri, de $29 \pm 5$ días de posparto. Se determinó el porcentaje de polimorfonucleares (PMN) con relación al número total de células presentes en las muestras de CE. Con la ultrasonografía se determinó la ecotextura uterina, presencia de contenido uterino y el diámetro de los cuernos uterinos. Posterior a la evaluación uterina se realizó el empadre controlado y 35 días después el diagnóstico de preñez por US. Se observó un mayor porcentaje de PMN $(\mathrm{p}<0.05)$ en las muestras de citología endometrial obtenidas con la técnica del citocepillo en comparación al lavado uterino. Los cuernos uterinos tuvieron una ecotextura heterogénea y sin presencia de contenido. El diámetro del cuerno uterino izquierdo fue mayor que el del cuerno uterino derecho $(\mathrm{p}<0.05)$. No hubo relación del porcentaje de PMN con diámetro de cuerno uterino ni con preñez. Según los resultados obtenidos, la CE no permitiría valorar adecuadamente la salud uterina, pues habría otros factores involucrados en una mayor respuesta quimiotáctica de los PMN que promueven su migración hacia útero. La ultrasonografía permitió una valoración objetiva de los cuernos uterinos y de la salud uterina, y permitió realizar el diagnóstico temprano de preñez.
\end{abstract}

Palabras clave: alpaca; citología endometrial; ultrasonografía; preñez

${ }^{1}$ Escuela Profesional de Medicina Veterinaria y Zootecnia, Facultad de Medicina Veterinaria y Zootecnia, Universidad Peruana Cayetano Heredia, Lima, Perú

${ }^{2}$ Centro de Investigación y Producción Chuquibambilla, Facultad de Medicina Veterinaria y Zootecnia, Universidad Nacional del Altiplano, Puno, Perú

${ }^{3}$ Departamento de Nutrición, Facultad de Zootecnia, Universidad Nacional Agraria La Molina, Lima, Perú

${ }^{4}$ E-mail: hugo.deza@upch.pe

Recibido: 14 de junio de 2018

Aceptado para publicación: 5 de septiembre de 2019 
The aim of this study was to evaluate the uterine health of alpacas by endometrial cytology (EC) using the cytobrush and uterine lavage techniques, to evaluate the uterine characteristics by ultrasound (US) and to relate them to the pregnancy rate. Multiparous $(n=20)$ and primiparous (20) Suri alpacas of $29 \pm 5$ days postpartum were used. The percentage of polymorphonuclear (PMN) was determined in relation to the total number of cells present in the EC samples. The uterine ecotexture, presence of uterine content and the diameter of the uterine horns were determined by ultrasonography. Subsequent to the uterine evaluation, controlled breeding was performed, and pregnancy diagnosis was done 35 days later by US. A higher percentage of PMN $(\mathrm{p}<0.05)$ was observed in endometrial cytology samples obtained with the cytobrush technique compared to uterine lavage. The uterine horns had a heterogeneous ecotexture and no presence of content. The diameter of the left uterine horn was greater than that of the right uterine horn $(\mathrm{p}<0.05)$. There was no relationship between percentage of PMN and uterine horn diameter or pregnancy. Based on the results obtained, the EC would not allow an adequate assessment of uterine health, as there would be other factors involved in a greater chemotactic response of the PMN that promote their migration to the uterus. Ultrasonography allowed an objective assessment of uterine horns and uterine health and allowed early diagnosis of pregnancy.

Key words: alpaca; endometrial cytology; ultrasonography; pregnancy

\section{INTRODUCCIÓN}

El Perú posee la mayor población de alpacas, cuyo hábitat natural se encuentra en los altos Andes por encima de los $4000 \mathrm{~m}$ de altura; siendo para la mayor parte de sus criadores la única fuente de sustento económico y la principal fuente de proteína en su dieta (Huanca et al., 2007). Uno de los inconvenientes en su crianza es la baja tasa de fertilidad (Bravo et al., 1995; Tibary et al., 2006), que podría estar relacionada con el alto porcentaje de hembras que quedan vacías (14$54 \%$ ) después de un periodo de empadre (Sapana et al., 2012), y la mortalidad embrionaria que se produciría en los primeros 30 días de gestación (Fernández-Baca et al., 1970; Bravo y Sumar, 1985; Tibary et al., 2006). Sin embargo, la baja eficiencia reproductiva podría también estar relacionada con posibles infecciones uterinas (Tibary y Anouassi, 2001).
La endometritis subclínica no manifiesta signos clínicos visibles; sin embargo, afecta significativamente el desempeño reproductivo de los animales (Kasimanickam et al., 2004; Gilbert et al., 2005), debido a la inflamación superficial del endometrio (Bondurant, 1999). El examen clínico reproductivo es frecuente en animales domésticos con el fin de evaluar el estado de salud uterina previa al servicio, siendo la citología endometrial un medio de diagnóstico aceptado (Kasimanickam et al., 2005). La presencia de células inflamatorias en el útero, como los polimorfonucleares (PMN), se asocia directamente a una posible infección (Barlund et al., 2008). Para el caso de camélidos se ha reportado que la presencia de $3-5 \%$ de neutrófilos en la muestra uterina sería indicativo de endometritis (Tibary y Anouassi, 2001).

La biopsia endometrial es una herramienta de diagnóstico definitivo de endometritis; sin embargo, es tediosa y cos- 
tosa (Gilbert et al., 2005; Sheldon et al., 2006). Se ha venido empleando métodos menos invasivos y de procedimientos más sencillos para la obtención de células endometriales, como el citocepillo y el lavado uterino (Tibary y Anouassi, 2001; Kasimanickam et al., 2005; Gilbert et al., 2005) con excelentes resultados, y que han servido como estándares de comparación con otras técnicas de diagnóstico como la ultrasonografía (Barlund et al., 2008). Esta última es también una herramienta útil para el diagnóstico de condiciones patológicas que pueden comprometer la fertilidad y la salud uterina (Gonzalez-Bulnes et al., 2010); sin embargo, el nivel de concordancia entre la citología y la ultrasonografía es aún baja (Kasimanickam et al., 2004).

Por lo general, los investigadores están de acuerdo en que la endometritis tiene efectos perjudiciales sobre el comportamiento reproductivo (Gilbert et al., 2005). No obstante, se han realizado pocos estudios en camélidos que evalúen la salud uterina, así como los métodos de diagnóstico y citología uterina; por tal razón, el presente experimento tuvo como objetivo evaluar el estado de salud uterina previa al servicio por los métodos de citología endometrial y ultrasonografía.

\section{Materiales y Métodos}

\section{Ubicación del Estudio y Animales}

El estudio se realizó en el Centro de Investigación y Producción Chuquibambilla (Umachiri, Melgar, Puno, ubicado a 3974 $\mathrm{msnm}$ ), entre enero y marzo de 2016. Alpacas Suri con cría, multíparas $(\mathrm{n}=20)$ y primíparas $(\mathrm{n}=19)$, clínicamente normales y sin presencia de descargas vaginales anormales fueron incluidas en el estudio. La ultrasonografía se realizó antes de la toma de muestras de citología endometrial por las técnicas de citocepillo y lavado uterino.

\section{Ultrasonografía}

La ultrasonografía y toma de muestras se realizaron a los $29 \pm 5$ días posparto. Para esto, los animales fueron sujetados en posición decúbito ventral sobre una tarima a una altura de $30 \mathrm{~cm}$ respecto del piso. Las imágenes de ultrasonografía fueron obtenidas con el modo B/B a tiempo real realizadas con un escáner de ultrasonido (WED 3100, China), conectado a un transductor lineal de $6.5 \mathrm{MHz}$, adecuando el procedimiento descrito por You et al. (2013). Se determinaron las características ecográficas cualitativas de ecotextura y contenido uterino y el diámetro de cuernos uterinos (en $\mathrm{mm}$ ). El tiempo de evaluación por animal varió entre 5 y 7 minutos. Todas las evaluaciones fueron realizadas por el mismo operador.

\section{Citocepillo}

Las muestras endometriales para la técnica del citocepillo fueron colectadas adaptando el procedimiento descrito por Kasimanickam et al. (2005). Brevemente, se utilizó un citocepillo de uso humano, cortado a $5 \mathrm{~cm}$ de la punta del cepillo y se insertó en un aplicador Cassou 0.25 previamente calentado sobre un mechero. El cepillo fue cubierto por una funda para inseminación y protegido por una camiseta sanitaria. Una vez que la alpaca estaba inmovilizada en posición decúbito ventral, sin heces en el recto y la zona perianal lavada y desinfectada, se introdujo el equipo con el citocepillo por el canal vaginal, guiado mediante palpación rectal hasta el útero, donde el citocepillo fue expuesto y girado $360^{\circ}$ en sentido de las agujas del reloj. Luego, se le retrajo hacia la funda de inseminación para ser retirado.

Con los citocepillos conteniendo las muestras se prepararon dos frotis por hembra en láminas portaobjetos. Las láminas fueron secadas a medio ambiente. Una de las láminas fue teñida con Giemsa (Giemsa stain, 
Cuadro 1. Diámetro de los cuernos uterinos de alpacas primíparas y multíparas al momento del servicio (promedio \pm error estándar)

\begin{tabular}{lccc}
\hline & $\begin{array}{c}\text { Diámetro de cuerno } \\
\text { uterino, mm }\end{array}$ & Rango, mm & $\begin{array}{c}\text { Coeficiente de } \\
\text { variación, } \%\end{array}$ \\
\hline Cuerno uterino & & & \\
Derecho & $15.95 \pm 0.50^{\mathrm{b}}$ & $10.0-22.0$ & 22.91 \\
Izquierdo & $17.89 \pm 0.68^{\mathrm{a}}$ & $11.0-26.0$ & 23.45 \\
Número de parto & & \\
$\quad$ Primípara & $16.47 \pm 0.63^{\mathrm{a}}$ & $12.0-26.0$ & 23.39 \\
Multípara & $17.37 \pm 0.68^{\mathrm{a}}$ & $10.0-24.0$ & 24.18 \\
Interacción cuerno uterino & & & \\
x número de parto & & & \\
Derecho - Primípara & $15.16 \pm 0.66^{\mathrm{a}}$ & $12.0-22.0$ & 18.95 \\
Izquierdo - Primípara & $17.79 \pm 0.99^{\mathrm{a}}$ & $12.0-26.0$ & 24.26 \\
Derecho - Multípara & $16.74 \pm 0.97^{\mathrm{a}}$ & $10.0-22.0$ & 25.26 \\
Izquierdo - Multípara & $18.00 \pm 0.96^{\mathrm{a}}$ & $11.0-24.0$ & 23.28 \\
\hline
\end{tabular}

$a, b$ Letras diferentes dentro de columnas indican valores significativamente diferentes $(p<0.05)$

Sigma Aldrich $\left.{ }^{\circledR}\right)$ y la otra con la tinción Diff Quik (Differential Quik Stain kit, Polysciences Inc $($ ), siguiendo el protocolo establecido por cada laboratorio. Las láminas fueron leídas en un microscopio óptico a 400X. Se hizo el recuento de un mínimo de 100 células entre PMN y células uterinas, y se determinó la proporción de PMN respecto de la cuenta total.

\section{Lavado Uterino}

Se adecuó la técnica descrita por Dini et al. (2015). Brevemente, se utilizó una sonda Foley 14Fr estéril, a la que se insertó el mandril del aplicador de Cassou para darle rigidez. El catéter fue cubierto con una camiseta sanitaria. En la hembra, una vez sujeta e higienizada, se introdujo el catéter en el canal vaginal, guiado mediante palpación rectal, y una vez que la punta del catéter estuvo dentro del útero se fijó la sonda insuflando $5 \mathrm{ml}$ de aire a su balón de goma. Para el lavado uterino se infundió $15 \mathrm{ml}$ de fosfato buffer salino 1X (PBS; Sigma P3813) dentro de la cavidad uterina, se realizó un masaje de los cuernos uterinos y se procedió a recuperar el líquido infundido en tubos cónicos de $15 \mathrm{ml}$.

Las muestras fueron centrifugadas a 600 $\mathrm{x} g$ por 15 min, se decantó el líquido y del pellet del fondo del tubo se aspiró $10 \mu 1$. Se hicieron dos frotis por animal.

\section{Empadre Controlado}

Luego de la toma de muestras se realizó el empadre controlado de las alpacas. Se utilizó un corral de $200 \mathrm{~m}^{2}(10 \times 20 \mathrm{~m})$ donde se introdujeron las hembras en grupos de 10 animales y dos machos para que realicen el cortejo. Estos machos no fueron los que realizaron las montas. Se consideraron receptivas a las hembras que tomaron la posición de cópula antes de los 2 minutos de cortejo.

Las hembras receptivas fueron llevadas a un corral contiguo $(15 \times 20 \mathrm{~m})$, para el empadre controlado con machos de fertilidad comprobada. Se utilizaron 15 machos para 


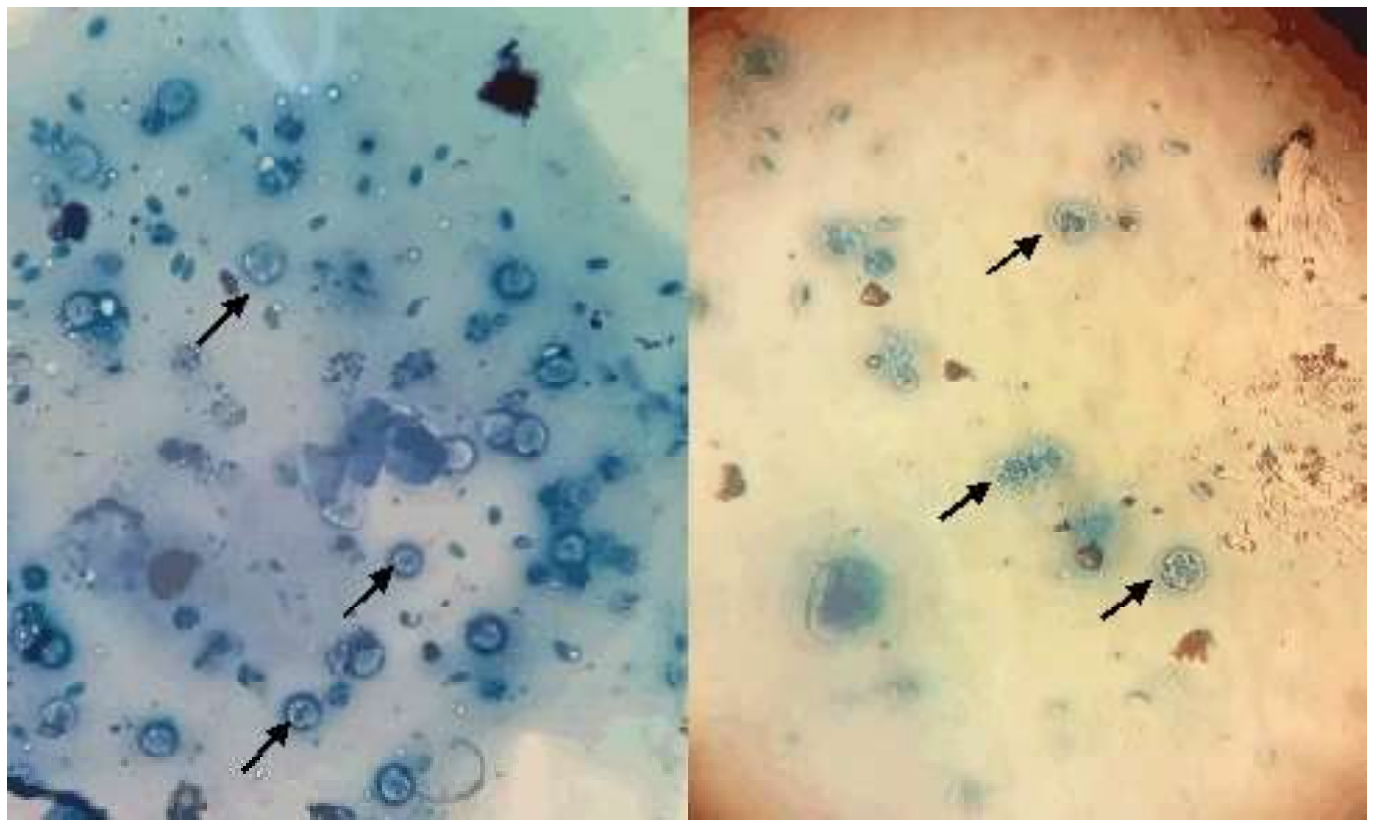

Figura 1. Polimorfo nucleares PMN $(\rightarrow)$ en lámina de citología endometrial. Izquierda: muestra tomada con citocepillo y procesada con tinción Giemsa; Derecha: muestra tomada por lavado uterino y procesada con tinción Diff Quik

el empadre de las hembras. Los machos realizaron solo una monta por día y con una frecuencia inter diaria. Se registró el tiempo de cópula. El diagnóstico de preñez se realizó por ultrasonografía a los 35 días del servicio. Las imágenes ultrasonográficas proyectaron la presencia (preñada) o ausencia (vacía) de la vesícula embrionaria.

\section{Análisis Estadístico}

Se utilizó un diseño completamente al azar (DCA) con un arreglo factorial de $2 \times 2$ (número de parto [primípara, multípara] y cuerno uterino [izquierdo, derecho) para la evaluación del diámetro de la pared uterina. Los porcentajes de neutrófilos fueron convertidos a valores angulares usando la transformación arco seno y luego sometidos a la prueba de Kolmogorov-Smirnov para determinar la normalidad y homosedasticidad con un $95 \%$ de confianza. Estos valores fueron analizados con un DCA con un arreglo factorial de $2 \times 2 \times 2$ (número de parto [primípara, multípara] x técnica empleada [citocepillo, lavado uterino] x técnica de tinción [Giemsa, Diff Quik). La prueba de medias se realizó con la prueba de Tukey y se determinó diferencia significativa cuando $\mathrm{p}<0.05$.

\section{Resultados}

El diámetro de los cuernos uterinos se muestra en el Cuadro 1. El diámetro del cuerno uterino izquierdo fue mayor, tanto en primíparas como multíparas $(\mathrm{p}<0.05)$ en comparación con el cuerno uterino derecho. No hubo diferencias significativas en el diámetro promedio de los cuernos uterinos entre primíparas y multíparas. La ecotextura observada de los cuernos uterinos previo al servicio fue heterogénea, tanto en primíparas como en multíparas, sin que se haya observado presencia de contenido uterino. 
Cuadro 2. Recuentos de polimorfonucleares (PMN) de muestras uterinas previo al servicio ( $29 \pm 5$ días posparto), según el método de colección de la muestra y de la tinción, en alpacas primíparas y multíparas

\begin{tabular}{lcc}
\hline & $\begin{array}{c}\text { Media, } \\
\%\end{array}$ & $\begin{array}{c}\text { Valor } \\
\text { máximo, } \\
\%\end{array}$ \\
\hline $\begin{array}{l}\text { Número de parto } \\
\text { Multípara (M) }\end{array}$ & $13.7^{\text {a }}$ & $0-57.2$ \\
Primípara (P) & $10.9^{\mathrm{a}}$ & $0-69.4$ \\
Técnica de obtención de & & \\
muestras & & \\
Citocepillo (CB) & $19.1^{\mathrm{a}}$ & $0-69.4$ \\
Lavado uterino & $4.7^{\mathrm{b}}$ & $0-30.6$ \\
(LU) & & \\
Técnica de tinción & & \\
Diff Quik (DQ) & $11.6^{\mathrm{a}}$ & $0-56.6$ \\
Giemsa (G) & $13.0^{\mathrm{a}}$ & $0-69.4$ \\
Interacciones & & \\
P-CB-DQ & $12.9^{\mathrm{a}}$ & $0-56.6$ \\
P-CB-G & $16.0^{\mathrm{a}}$ & $0-69.4$ \\
P-LU-DQ & $6.8^{\mathrm{a}}$ & $0-30.4$ \\
P-LU-G & $7.1^{\mathrm{a}}$ & $0-30.6$ \\
M-CB-DQ & $23.0^{\mathrm{a}}$ & $1.0-55.4$ \\
M-CB-G & $24.6^{\mathrm{a}}$ & $1.6-57.2$ \\
M-LU-DQ & $2.5^{\mathrm{a}}$ & $0-12.8$ \\
M-LU-G & $2.5^{\mathrm{a}}$ & $0-13.8$ \\
\hline a,b Letras diferentes dentro de & columnas y \\
variables indican valores & significativamente \\
diferentes (p<0.05) & & \\
& &
\end{tabular}

Los resultados de las lecturas de las muestras de citología endometrial previo al servicio indican mayor porcentaje de PMN en las muestras obtenidas con la técnica de citocepillo respecto de la técnica de lavado uterino $(\mathrm{p}<0.05)$. Por otro lado, el número de parto y el método de tinción de las muestras no tuvieron efecto significativo sobre los recuentos de PMN (Cuadro 2).

No hubo diferencia significativa en el porcentaje de PMN en las muestras de citología endometrial ni en el diámetro de los cuernos uterinos al ser comparados entre alpacas que concibieron y aquellas que quedaron vacías; sin embargo, se pudo observar una tendencia a un mayor porcentaje de PMN y un menor diámetro de los cuernos uterinos en alpacas que llegaron a concebir.

\section{Discusión}

La diferencia en el diámetro de los cuernos uterinos observados en la ecografía no necesariamente es indicativa de inflamación uterina. El incremento del tamaño puede reflejar daño físico, pero también varía por efecto de la raza, edad y nivel nutricional (Sheldon et al., 2006). Más aún, esta diferencia no solo obedecería al hecho de que la gestación en la alpaca se da en el $98 \%$ de los casos en el cuerno uterino izquierdo (Bravo et al., 1995; Ferrer, 2014), sino más bien, a que esta diferencia se da desde la etapa fetal (Mendoza et al., 2013).

La diferencia en diámetro entre los cuernos uterinos observados en este estudio es menor a la reportada en estudios post morten $(3.08 \pm 0.54 \mathrm{~cm}$, Mendoza et al., 2013), posiblemente como consecuencia de las técnicas utilizadas para estimar el diámetro de los cuernos uterinos. Es factible observar diferencias de hasta $1 \mathrm{~cm}$ entre la valoración por ultrasonografía y otros métodos como la palpación rectal en ganado bovino (Sheldon y Owens, 2017)

La ecotextura heterogénea de los cuernos uterinos de las alpacas puede estar relacionada con los niveles séricos de estradiol, que se mantienen lo suficientemente altos como para propiciar la receptividad sexual de las hembras (Vaughan, 2011). Tales concentraciones en otras especies y en camélidos pueden producir edematización e inflamación de los cuernos uterinos (Gonzalez-Bulnes et al., 2010; Rodriguez et al., 2014), generando una apariencia ultrasonográfica pobremente definida y predominantemente hipoecogénica (Gonzalez-Bulnes et al., 2010). 
La ausencia de contenido uterino no sólo indicaría que, en el caso de multíparas, la involución uterina fue completa al momento de la evaluación ultrasonográfica, hecho que estuviera acorde con reportes previos (Bravo et al., 1995; Tibary y Anouassi, 2001), sino además, haría suponer la ausencia de un proceso inflamatorio en útero (Sheldon y Owens, 2017), pues la presencia de contenido uterino está directamente relacionada con desarrollo bacteriano (Mateus et al., 2002).

Las diferencias en el porcentaje de PMN entre las técnicas de citocepillo y lavado uterino obedecería a las diferencias en los procedimientos de cada una de ellas. El citocepillo toma muestras directamente de la pared del cuerno uterino a través de un raspado suave, mientras que el lavado uterino recupera las células que se desprenden de la pared uterina tras la infusión de la solución de lavado en el útero. Por otro lado, la obtención de un mayor número de células, que además se encuentren bien preservadas, es indicativo de una mayor eficiencia de la técnica, haciéndola adecuada para realizar un diagnóstico (Barlund et al., 2008). En el presente experimento, el citocepillo ofrecería un mejor diagnóstico y, posiblemente, los resultados podrían ser más repetibles (Kasimanickam et al., 2005). Además, se caracteriza por ser una prueba rápida, especifica, sensible y económica, lo que la convierte en una herramienta valiosa para la investigación sobre el rol y la importancia de la endometritis (Gilbert et al., 2005). No obstante, se debe considerar que la técnica de lavado uterino tiene por ventaja la recuperación de células de una mayor superficie de área de la luz uterina, de allí que podría proveer una muestra más representativa del contenido uterino (Bonnett et al., 1991; Kasimanickam et al., 2005).

Respecto a las técnicas de tinción, tanto Diff Quik como Giemsa generaron una adecuada coloración de las células endometriales; así mismo, proveen una buena nitidez de las células al momento de ser observadas al microscopio (Figura 1), hecho también reportado en bovinos (Kasimanickan et al., 2004; Gilbert et al., 2005; Melcher et al., 2014; Pascottini et al., 2015, Pascottini y Opsomer, 2016). Sin embargo, según Pascottini et al. (2015), más del 90\% de los estudios utilizan la técnica de tinción DiffQuik (Wright - Giemsa modificada) por su practicidad y fiabilidad.

Para el diagnóstico de endometritis en camélidos, Sandoval et al. (2015) y Tibary y Anousassi (2001) señalan que la presencia de 3-5\% de neutrófilos PMN en muestras de citología endometrial en alpacas indican la ocurrencia de endometritis. De acuerdo con ello, un alto porcentaje de alpacas, especialmente cuando las muestras son tomadas con citocepillo $(82.50 \%)$ serían positivas a endometritis; sin embargo, los resultados de la evaluación ultrasonográfica no se relacionan con los porcentajes de PMN, dado que no se apreció contenido uterino y las tasas de preñez fueron similares entre alpacas con alto (e» $3 \%)$ y bajo porcentaje $(<3 \%)$ de PMN. Esto podría sugerir que el porcentaje de PMN en muestras de citología endometrial podría tener otro rol en el mantenimiento de la salud uterina en alpacas, aspecto que requiere ser investigado más ampliamente. La baja relación entre el porcentaje de PMN y la ultrasonografía $\left(\mathrm{r}^{2}=0.02\right)$ también fue observada por Kasimanickam et al. (2005) en vacas, posiblemente a consecuencia de que estas técnicas miden dos factores causales diferentes (la citología endometrial mide la respuesta celular y la ultrasonografía mide el grosor y contenido del útero).

Los resultados del presente experimento muestran un considerable porcentaje de PMN en útero, y si se considera que su presencia en el ambiente uterino es la primera respuesta del sistema inmune innato frente a una infección bacteriana, dicho incremento podría reflejar una endometritis subclínica, tal y como se considera en vacunos y ovinos (Gabler et al., 2010); sin embargo, esta movilización de los PMN en alpacas podría deberse a efectos endocrinos, debido a que la quimiotáxis es afectada por el momento 
Cuadro 3. Citología endometrial (polimorfonucleares [PMN]) y diámetro de cuernos uterinos alpacas previos al servicio que resultaron preñadas y vacías

\begin{tabular}{llcc}
\hline Técnica de evaluación & & Preñadas & Vacías \\
\hline Lavado uterino, \%PMN & Media & $7.07^{\mathrm{a}}$ & $4.04^{\mathrm{a}}$ \\
& Rango & $0-30.40$ & $0-30.60$ \\
Citocepillo, \%PMN & Media & $27.10^{\mathrm{a}}$ & $13.40^{\mathrm{a}}$ \\
& Rango & $4.20-69.40$ & $0-37.20$ \\
Diámetro uterino, mm & Media \pm EE & $16.71 \pm 0.85^{\mathrm{a}}$ & $17.30 \pm 0.72^{\mathrm{a}}$ \\
& Rango & $10.50-22.50$ & $13.00-22.50$ \\
Alpacas con $\geq 3 \%$ de PMN & Porcentaje & $52.0^{\mathrm{a}}$ & $48.0^{\mathrm{a}}$ \\
\hline
\end{tabular}

a Superíndices iguales dentro de filas indican ausencia de significancia estadística ( $p>0.05)$

posparto en el que se evalúa a la hembra y a las etapas del ciclo estral. Gabler et al. (2010) reportaron un pico en la proporción de PMN entre los 10 y 24 días posparto en vacas, hecho que fue relacionado al incremento en la expresión RNAm de muchos factores proinflamatorios y sustancias quimioatrayentes secretadas por las células epiteliales, las que dirigen a los PMN al lugar de inflamación.

Esto haría suponer que la regulación de los PMN y la infiltración de los mismos en el útero de las alpacas no necesariamente obedecería a un proceso inflamatorio como la endometritis subclínica; sino podría ser efecto de una sinergia entre los eventos posparto que se acompaña de cambios endocrinos y metabólicos, los que modulan la capacidad fagocítica y la expresión genética de los PMN (Sheldon et al., 2009). Dada la particularidad reproductiva de la alpaca, que constantemente tiene un folículo capaz de producir suficientes niveles de estrógenos y siendo estos los que jugarían un rol importante en la modulación de la respuesta quimiotáctica de los PMN, tal vez una proporción mayor al 3\% de estas células en las muestras de citología endometrial no implicaría la ocurrencia de una endometritis subclínica y como tal no afectarían las tasas de preñez, tal como se observó en el presente experimento.

\section{Conclusiones}

- La citología endometrial no sería de ayuda diagnóstica en la evaluación la salud uterina, pues habría otros factores involucrados en una mayor respuesta quimiotáctica de los PMN, que promueven su migración hacia útero.

- La ultrasonografía permite una mejor valoración de la salud uterina, además de ser muy eficiente en el diagnóstico temprano de preñez.

\section{Literatura Citada}

\section{Barlund CS, Carruthers TD, Waldner} CL, Palmer CW. 2008. A comparison of diagnostic techniques for postpartum endometritis in dairy cattle. Theriogenology 69: 714-723. doi: 10.1016/ j.theriogenology.2007.12.005 
2. Bondurant RH. 1999. Inflammation in the bovine female reproductive tract. J Anim Sci 77(Suppl 2): 101-110. doi: 10.2527/1999.77suppl_2101x

3. Bonnett BN, Miller RB, Etherington WG, Martin SW, Johnson WH. 1991. Endometrial biopsy in Holstein-Frisian dairy cows I. Technique. Histological criterial and results. Can J Vet Res 55: 155-161. doi: 10.1016/j.theriogenology.2007.12.005

4. Bravo W, Sumar J. 1985. Factores que determinan la fertilidad en alpacas. En: Anales V Convención Internacional sobre Camélidos Sudamericanos. Cusco, Perú.

5. Bravo WP, Lasley B, Fowler M. 1995. Resumption of ovarian follicular activity y uterine involution in the postpartum llama. Theriogenology 44: 783-791. doi: 10.1016/0093-691X(95)00265-A

6. Dini P, Farhoodi M, Hostens M, Van Eetvelde M, Pascottini OB, Fazeli MH, Opsoner G 2015. Effect of uterine lavage on neutrophil counts in postpartum dairy cows. Anim Reprod Sci 158: 2530. doi: 10.1016/j.anireprosci.2015.04.005

7. Fernandez-Baca S, Hansel W, Novoa C. 1970. Embryonic mortality in the alpaca. Biol Reprod 3: 243-252. doi: 10.1093/biolreprod/3.2.243

8. Ferrer MS. 2014. Diagnosis of pregnancy and evaluation of high-risk pregnancy. In: Llama and alpaca care. Canada: Elsevier. p 250-256.

9. Gabler C, Fischer C, Drillich M, Einspanier R, Heuwieser W. 2010. Time-dependent mRNA expression of selected pro-inflammatory factors in the endometrium of primiparous cows postpartum. Reprod Biol Endocrin 8: 152. doi: 10.1186/1477-7827-8-152

10. Gilbert RO, Shin ST, Guard CL, Erb HN, Frajblat M. 2005. Prevalence of endometritis y its effects on reproductive performance of dairy cows. Theriogenology 64: 1879-1888. doi: 10.1016/ j.theriogenology.2005.04.022

11. Gonzalez-Bulnes A, Pallares P, Vazquez MI. 2010. Ultrasonographic imaging in small ruminant reproduction.
Reprod Domest Anim 45(Suppl 2): 9-20. doi: 10.1111/j.1439-0531.2010.01640.x

12. Huanca T, Apaza N, Sapana R. 2007. Defectos congénitos y hereditarios visibles en alpacas de dos zonas representativas de la Región Puno. En: XX Reunión de la Asociación Latinoamericana de Producción Animal. Cusco, Perú.

13. Kasimanickam R, Duffield TF, Foster $R A$, Gartley CJ, Leslie KE, Walton JS, Johnson WH. 2004. Endometrial cytology y ultrasonography for the detection of subclinical endometritis in postpartum dairy cows. Theriogenology 62: 9-23. doi: 10.1016/j.theriogenology.2003.03.001

14. Kasimanickam R, Duffield TF, Foster $R A$, Gartley CJ, Leslie KE, Walton JS, Johnson WH. 2005. A comparison of the cytobrush and uterine lavage techniques to evaluate endometrial cytology in clinically normal postpartum dairy cows. Can Vet J 46: 255-259.

15. Mateus L, Lopes da Costa L, Bernardo F, Robalo Silva J. 2002. Influence of puerperal uterine infection on uterine involution y postpartum ovarian activity in dairy cows. Reprod Domest Anim 37: 31-35. doi: 10.1046/j.1439-0531.2002.00317.x

16. Melcher Y, Prunner I, Drillich M. 2014. Degree of variation y reproducibility of different methods for the diagnosis of subclinical endometritis. Theriogenology 82: 57-63. doi: 10.1016/ j.theriogenology.2014.03.003

17. Mendoza G, Echevarría L, Llerena C, Castro A, Domínguez M, Gómez S, Ghezzi M, et al. 2014. Comparación morfológica entre el útero fetal y el útero adulto de la alpaca (Vicugna pacos) y la llama (Lama glama). Salud Tecnol Vet 1: 1-6.

18. Pascottini OA, Dini P, Hostens M, Ducatelle M, Opsomer G 2015. A novel cytologic sampling technique to diagnose subclinical endometritis y comparison of staining methods for endometrial cytology samples in dairy cows. Theriogenology 84: 1438-1446. doi: 10.1016/j.therioge-nology.2015.-07.032 
19. Pascottini OA, Opsomer G. 2016. Postpartum uterine diseases in dairy cows: a review with emphasis on subclinical endometritis. Vlaams Diergen Tijds 85: 378-385.

20. Rodriguez JK, Pearson LK, Tibary A. 2014. Clinical examination of the female reproductive function. In: Llama and alpaca care. Canada: Elsevier. p 168-187.

21. Sandoval R, Ruiz L, Santiani A, Delgado A, Choez K, Pizarro J, Arévalo I. 2015. Evaluación de la capacidad diagnóstica de dos pruebas de campo en la detección de infecciones uterinas en camélidos sudamericanos. Spermova 5: 144-148.

22. Sapana R, Huanca T, Cárdenas O, Mamani R, González M, Apaza N. 2012. Empadre controlado de alpacas Huacaya del CIP Quimsachata de INIA-Puno-Perú. En: VI Congreso Mundial sobre Cámelidos. Arica, Chile.

23. Sheldon IM, Owens SE. 2017. Postpartum uterine infection and endometritis in dairy cattle. Anim Reprod 14: 622-629. doi: 10.21451/1984-3143AR1006

24. Sheldon IM, Cronin J, Goetze L, Donofrio G, Schuberth HJ. 2009. Defining postpartum uterine disease and the mechanisms of infection and immunity in the female reproductive tract in cattle. Biol Reprod 81: 1025-1032. doi: 10.1095/biolreprod.109.077370

25. Sheldon IM, Lewis GS, LeBlanc S, Gilbert RO. 2006. Defining postpartum uterine disease in cattle. Theriogenology 65: 1516-1530. doi: 10.1016/j.theriogenology.2005.08.021

26. Tibary A, Anouassi A. 2001. Uterine infections in camelidae. Vet Sci Tomorrow 1: 1-12.

27. Tibary A, Fite C, Anouassi A, Sghiri A. 2006. Infectious cause of reproductive loss in camelids. Theriogenology 66 : 633-647. doi: 10.106/j.theriogenology.2006.04.008

28. Vaughan J. 2011. Ovarian function in South American camelids (alpacas, 1lamas, vicunas, guanacos). Anim Reprod Sci 124: 237-243. doi: 10.1016/ j.anireprosci.2010.08.031

29. You R, Lv L, Cheng Z, He J, Smith GW, Dong Ch. 2013. Application of ultrasonography for early pregnancy diagnosis in alpacas (Lama pacos). $\mathrm{J}$ Anim Vet Adv 12: 539-543. doi: 10.3923/ javaa.2013.539.543 